\title{
Analogical Reasoning in a Capuchin Monkey (Cebus apella)
}

\author{
Erica Hoy Kennedy and Dorothy M. Fragaszy \\ University of Georgia
}

\begin{abstract}
Previous evidence has suggested that analogical reasoning (recognizing similarities among object relations when the objects themselves are dissimilar) is limited to humans and apes. This study investigated whether capuchin monkeys (Cebus apella) can use analogical reasoning to solve a 3 -dimensional search task. The task involved hiding a food item under 1 of 2 or 3 plastic cups of different sizes and then allowing subjects to search for food hidden under the cup of analogous size in their own set of cups. Four monkeys were exposed to a series of relational matching tasks. If subjects reached criterion on these tasks, they were exposed to relational transfer tasks involving novel stimuli. Three of the monkeys failed to reach criterion on the basic relational matching tasks and therefore were not tested further. One monkey, however, revealed above-chance performance on a series of transfer tasks with 3 novel stimuli. This evidence suggests that contrary to previous arguments, a member of a New World monkey species can solve an analogical problem.
\end{abstract}

Keywords: analogical reasoning, capuchin monkeys, Cebus apella

The ability to reason analogically is often considered one of the major hallmarks of human cognition. According to Anderson (2000, p. 247), analogy can be defined as "the process by which a problem solver maps the solution for one problem into a solution for another problem." The use of analogies makes categorization of new objects and solutions to novel problems more efficient because knowledge of similar objects and solutions can be used rather than attempting to solve problems or categorize items from scratch. The ability to reason analogically has clearly supported human advancement in science and technology and thus has been one of the keys to human progress. The purpose of this study was to determine the degree to which the capacity to understand and use analogies is present in a New World monkey species. By studying the ability of nonhuman primates to use analogies to solve problems, we may gain a better understanding of our own reasoning ability and of the evolutionary pathways that may have led to the appearance of analogical reasoning in humans.

Developmental psychologists have examined the factors that influence the time frame in which children are able to comprehend analogies, as analogical reasoning develops over time. It is argued that children initially respond to literal (physical) similarity between objects in terms of color, shape, and so forth (Gentner, Rattermann, Markman, \& Kotovsky, 1995). Gentner et al. (1995)

Erica Hoy Kennedy and Dorothy M. Fragaszy, Department of Psychology, University of Georgia.

We thank Irwin Bernstein, Jonathon Crystal, Adam Goodie and Roger K.R. Thompson for their comments and advice. Thanks also to those that helped throughout the course of testing, including Jessi Crast, Robin Cooley, Grace Fletcher, Tephi Jeyaraj, Steve Kennedy, Freya Liu, Carrie Rosengart and Liz Simpson. We acknowledge funding in part from National Institutes of Health Grant HD38051 to Georgia State University.

Correspondence concerning this article should be addressed to Erica Hoy Kennedy, who is now at the Department of Psychology, Frostburg State University, 101 Braddock Road, Frostburg, MD 21532. E-mail: ehkennedy@frostburg.edu argued that as children acquire greater knowledge, they go through a "relational shift" in which they gain the ability to understand relational similarities in addition to the physical object similarities on which they previously relied. To illustrate this shift, Gentner et al. gave the example of children asked to interpret the following metaphor: "A tape recorder is like a camera." In this case, 6-yearolds used object-based interpretations ("both are metal and black"), and 9-year-old children noticed the relational structure (the ability to record something for later; Gentner et al., 1995).

There are two primary explanations for the relational shift given in the literature. One explanation is that children undergo a global shift in their cognitive abilities. In this case, the ability to understand higher order relations is linked to Piaget's stages of cognitive development. Specifically, Piaget (1966) argued that children cannot complete relational analogies (non-object based) until they have reached the stage of formal operations at 11-12 years of age. An opposing explanation argues that children are able to focus on relational similarities as a function of their knowledge base (Gentner et al., 1995). According to this argument, younger children can perform analogies based on relational features if they involve a familiar context. In a 1977 study by Gentner, children of varying ages were asked, "If the tree had a knee, where would it be?" Children as young as 4 were able to solve this analogy involving relational features, suggesting that children do not need to reach the formal operations stage before they can reason in this way (Gentner, 1977).

If children are able to solve complex relational analogies as their knowledge increases, is it possible that other species with experience solving certain kinds of relational problems would also be able to solve similar, but novel, relational problems? It is argued that this may indeed be the case for chimpanzees. One chimpanzee in particular (Sarah) has shown much success in solving both functional and standard item analogies. In a 1981 study by Gillian, Premack, and Woodruff, Sarah was presented with a physical representation of an analogy in $2 \times 2$ matrix form. The analogy contained two relations, one between $\mathrm{A}$ and $\mathrm{A}^{\prime}$ and the other 
between $B$ and $B^{\prime}$. For example, Sarah was presented with a large blue triangle $(\mathrm{A})$, a small blue triangle $\left(\mathrm{A}^{\prime}\right)$, a large yellow crescent (B), and a small yellow crescent $\left(\mathrm{B}^{\prime}\right)$ in matrix form. Although the actual items being compared in this case were different (triangle and crescent), the relation between both pairs of items is the same (large to small). Sarah was presented with three stimuli in this form and was required to choose one item from a pair of objects to complete the analogy. One of the choices would complete a true analogy (i.e., maintain the same relationship between the pairs of objects), and the other item would not fit the analogy. It is important to note that the correct relation (large or small) varied across trials, so that it was not possible to use one generalized size rule (i.e., "always choose the smallest item") to solve these problems. Sarah was also exposed to analogies that required some understanding of the functional use of household objects that she had previously encountered. In this case, she was exposed to items such as a padlock and key in relation to a tin can and can opener. In previous experiments, Sarah had learned to represent the concepts same and different with plastic tokens (Oden, Thompson, \& Premack, 2001). In this particular study, Sarah was required to use her prior knowledge of these concepts to label whether the two pairs of presented objects represented a same or different relationship. Sarah was successful at these tasks in both the geometric and functional forms, implying that she has the capacity to reason at the more abstract analogical level (Oden et al., 2001).

It is possible that Sarah's ability to reason analogically was a function of her extensive training with the concepts of same and different (Premack \& Premack, 2003). Premack and Premack (2003) reported that chimpanzees without same-different training could solve basic relational matching problems as the result of extensive trial and error learning, although they were unable to solve more complex analogies. Thompson, Oden, and Boysen (1997) reported that chimpanzees with prior training with tokens were able to solve relational matching problems with nondifferential reinforcement, suggesting that the ability to use tokens facilitates analogical reasoning.

According to Oden et al. (2001), there is no evidence that primates other than apes are able to perceive or solve analogies. Studies investigating analogical reasoning in rhesus macaques (Macaca mulatta) and capuchin monkeys (Cebus apella) using conceptual matching-to-sample (MTS) and dishabituation paradigms have produced negative results in terms of the ability of these species to comprehend analogies (Thompson \& Oden, 1995; Thompson et al., 2000). These results suggested that there may be a fundamental difference between monkeys and apes in terms of their capacity to understand analogies.

A more recent study by Spinozzi, Lubrano, and Truppa (2004), however, provided evidence that monkeys are able to reason about abstract relations. In this study, five capuchins were tested on their ability to perform a MTS task that involved matching the spatial relations above and below. In this case, subjects were required to match an image of a horizontal line with a dot situated either above or below the line to another image representing the same spatial relationship (above or below) between the line and the dot. The monkeys were successful in matching the images when the choices included an image that matched the sample in terms of the relation between the line and the dot, but the distance between the dot and the line were varied so that there was no choice that was an identical match to the sample. The authors argued that these results provided evidence that capuchin monkeys can reason about abstract spatial relations (above vs. below). The current study aimed to determine whether capuchin monkeys were capable of solving a complex analogical task that more closely mirrored those that have been solved by young children and chimpanzees.

The capuchin subjects in the current study were chosen because they have had many years of experience solving experimental problems, particularly spatial relational problems such as navigating two-dimensional mazes and using a variety of tools (e.g., Cummins-Sebree \& Fragaszy, 2005; Fragaszy, Johnson-Pynn, Hirsh, \& Brakke, 2003; Johnson-Pynn, Fragaszy, Hirsh, Brakke, \& Greenfield, 1999; Rosengart \& Fragaszy, 2005; see Fragaszy \& Cummins-Sebree, 2005, for review). The developmental literature has provided much evidence suggesting that prior knowledge plays a key role in forming effective problem-solving strategies (Alexander \& Schwanenfluegel, 1994; Gaultney, Bjorklund, \& Schneider, 1992; Schneider, Bjorklund, \& Maier-Bruckner, 1996; Woloshyn, Pressley, \& Schneider, 1992). If the capuchins in this study were able to solve spatial analogies, it would suggest that analogical reasoning is not limited to apes, but instead that individuals from other taxa may also reason in this way, particularly if they have extensive experience solving relational problems.

This study was modeled from a task that has previously been used to test the analogical reasoning abilities of children. Gentner and Rattermann (1991) describe an unpublished study (Rattermann, Gentner, \& DeLoache, 1987) in which 3 and 4-year-old children were asked to complete what they called a "perceptual matching task". In this study, both the child and the experimenter had a set of three objects (such as flower pots) that decreased in size in a continuum from left to right. The experimenter would hide a sticker under one of her three objects and then ask the child to use this information to find a sticker hidden under the child's own set of objects. The correct response in all cases was to search under the object of the same relative size as that revealed by the demonstrator (i.e., smallest object to smallest object, or largest object to largest object, varying across trials). The purpose of the study was to determine whether the children would rely on a strategy dependent on object similarity or relational similarity. Thus, both sets of stimuli also included some degree of object similarity. For example, if the experimenter's set of stimuli included flower pots of sizes 4,3 , and 2 , then the child's set would include pots of sizes 3,2, and 1. Pots of size 3 and 2 occur in both sets (and are physically identical), but they do not share relational similarity between the sets (size 2 serves as the smallest for the experimenter, but as the middle size for the child). If children are able to use the rule based on relational similarity to find their sticker, then they should not be confused by the object similarity. Gentner and Rattermann (1991) report that younger children (3year-olds) showed poorer performance on this task in comparison with 4-year-olds, suggesting that the ability to reason about relations develops over time.

In the current study, capuchins with no prior training with symbols, the same-different concept, or MTS tasks were required to use the experimenter's demonstration of retrieving a hidden food item to find hidden food in the analogous location under the subject's set of objects. Although the basic task (searching for a hidden object) used in the current study was modeled after Rattermann et al.'s (1987) study with children (as described by Gentner \& Rattermann, 1991), several aspects of the procedure (such 
as number of trials and number and arrangement of stimuli) were altered to accommodate differences inherent in working with monkeys rather than children. A key difference was that the current study included a series of four different testing phases that were presented in an order that we hypothesized to increase in difficulty on the basis of the tasks' demands. This design feature was included in hopes of facilitating the emergence of analogical reasoning.

We expected that the extensive prior problem-solving experience of the subjects would provide the knowledge base needed for the monkeys to solve both physical matching and relational problems. In addition, we predicted that the specific experience with the physical matching task should aid the monkeys in acquiring the skills needed for the relational matching task. Previous work with these subjects (Hoy \& Fragaszy, 2004) revealed that capuchins that were exposed to computer mazes in order of increasing difficulty performed significantly better than those that received the same mazes in random order. This suggests that initial exposure to relatively simple problems (i.e., a physical matching task) may aid in the solution of more difficult problems presented later (relational problems).

We made two primary predictions concerning the performance of capuchins solving both physical identity and relational matching problems: (a) As previous research has shown that relational problems are more difficult than physical matching problems, subjects should require more trials to reach criterion for the relational matching task in comparison with the physical matching task and (b) capuchins should be able to reach criterion on both the relational matching task with the distracter and the relational transfer task if they reached criterion on the initial relational matching task (without the distracter). If capuchins are ultimately able to perform this task using a strategy based on relational similarity, then it can be argued that the ability to reason analogically is not limited solely to apes and humans and may instead be found in a more diverse range of species.

\section{Method}

\section{Subjects}

Four male capuchin monkeys (Cebus apella) participated in this study. These monkeys ranged in age from 10 to 20 years old. All subjects had several years of experience with instrumental spatial problem-solving tasks (including computer joystick problems and touchscreen and tool-use tasks; for more detailed descriptions of the previous experience of these subjects, see Cummins-Sebree \& Fragaszy, 2005; Fragaszy et al., 2003; Leighty \& Fragaszy, 2003; Rosengart \& Fragaszy, 2005). All subjects also had experience with a food search task as part of a study investigating the A-not-B error (Rosengart, 2005). The monkeys were pair-housed at the University of Georgia. None of the animals were food deprived during testing, although testing occurred before either their morning or afternoon feedings. The monkeys received a diet of Purina monkey chow and fruit. The care and experimental treatment of the monkeys followed local and federal regulations concerning humane care and treatment.

\section{Materials}

Subjects were transported to a room adjacent to their housing area for testing. Subjects were tested in a cage $(64 \mathrm{~cm} \times 47 \mathrm{~cm} \times$
$78 \mathrm{~cm}$ ) composed of metal mesh and two Plexiglas side panels. One of the Plexiglas panels contained a rectangular hole in the lower half of the panel that allowed subjects to extend their arms outside of the cage to manipulate objects. The stimuli were presented to the subjects on a metal cart that was pushed up to the Plexiglas panel of the test cage. This cart was the same height as the floor of test cage. A small steplike tiered platform was placed on top of the cart to display the stimuli. Both "steps" were 15.24 $\mathrm{cm} \times 20.32 \mathrm{~cm}$ in dimension and contained five evenly spaced markings to indicate the locations for stimulus placement. The second tier was approximately $2.54 \mathrm{~cm}$ higher than the bottom tier (see Figure 1). During testing, subjects were rewarded with a food item (dried fruit, nuts, or cereal) on completion of a successful search. A white poster board that was $60.56 \mathrm{~cm} \times 81.28 \mathrm{~cm}$ in dimension was used as a barrier to obstruct the subjects' view while the experimenter hid the food for each trial. A video camera was used to record testing sessions to allow playback for scoring purposes.

The experimental stimuli consisted of two sets of eight plastic stacking cups (children's toys), spray-painted black. The cups in these sets ranged in diameter from $5.08 \mathrm{~cm}$ to $8.89 \mathrm{~cm}$. A new set of stimuli was used for the final relational matching transfer testing phase. These stimuli consisted of two sets of eight plastic stacking cubes that were painted yellow and ranged in width from 4.13 to $7.30 \mathrm{~cm}$. Thus, these two stimulus sets differed in shape and color.

\section{Procedure}

Testing occurred over a span of approximately 9 months, during which time subjects were tested between 4 and 7 days per week. Each testing session lasted approximately $30 \mathrm{~min}$ and consisted of no more than 22 trials per day. This maximum number of trials per day was chosen because subjects' attention tended to decrease after 22 trials during preliminary training.

\section{Preliminary Training}

All subjects were exposed to a series of trials to familiarize them with the search task. For all phases, an assistant read aloud the size of the cups to be used in each trial, their position, and the relative size of the cup that was to serve as the hiding location ("big" or "small"). On the start of a trial, the experimenter called the subject's name to gain the subject's attention. For preliminary training, a single cup was placed on the bottom tier of the stimulus platform. The experimenter then lifted the cup and pointed to the food item hidden under it. The experimenter then once again covered the food with the cup, while keeping a finger on top of the cup hiding the food. The cart and platform were then pushed up to the test cage, allowing the subject to search under the cup and retrieve the food item. Until the monkey chose a cup, the experimenter fixed her gaze at a designated point on the wall above the testing cage. If the food was not retrieved in $30 \mathrm{~s}$, the trial was repeated. The size of the cup used in each trial was randomized, although no cup was used for more than two consecutive trials. Each subject was exposed to 20 initial training trials.

This same training procedure was then repeated using two cups of different sizes. In this case, the experimenter revealed a food item hidden under one of two cups that were placed side by side on the lower tier of the platform. The subject was then given imme- 

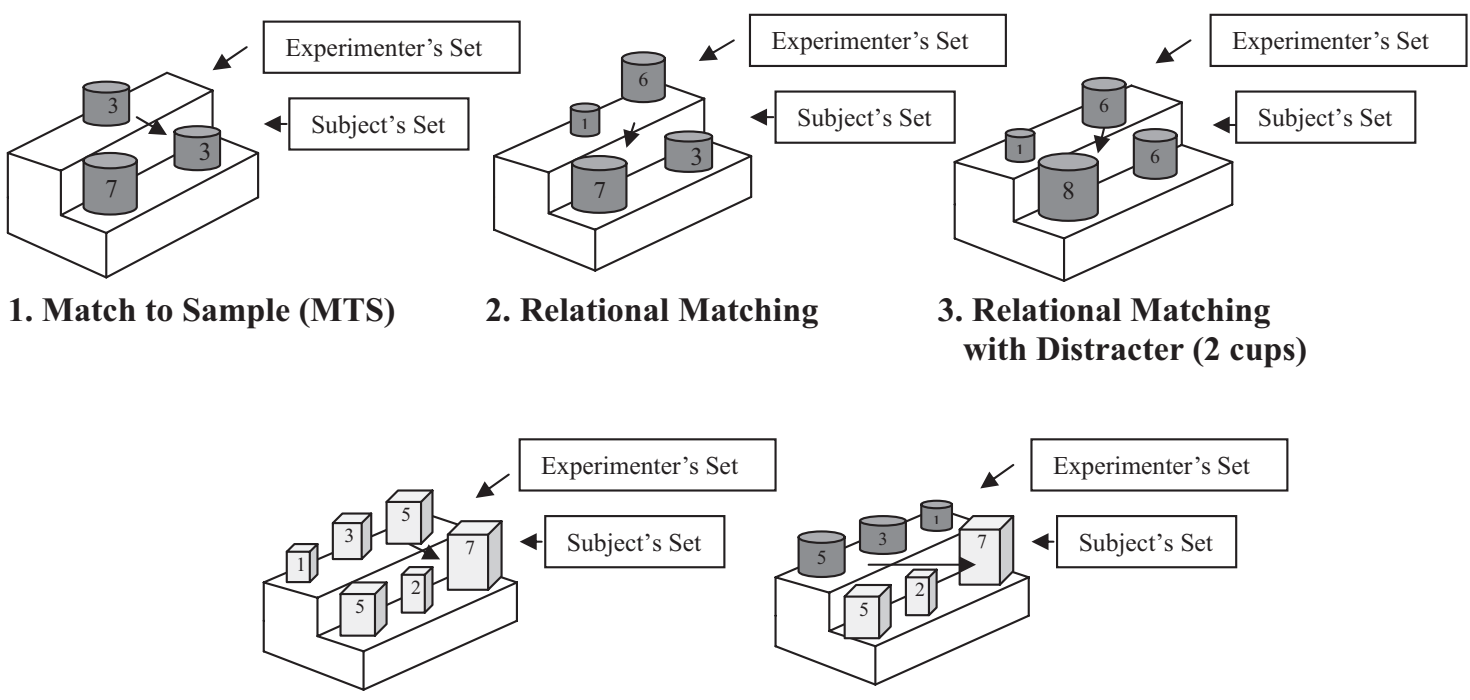

\section{Relational Matching with Distracter (Novel Stimuli)}

\section{Relational Matching with Distracter (Dissimilar Stimuli)}

Figure 1. Depiction of stimulus arrangement for five matching tasks. (Note that objects pictured are not drawn to scale.) The arrow between cups indicates the correct match.

diate access to the platform and allowed to search. In this training phase and all subsequent testing phases, if the subject chose the wrong cup, the cart was removed from the subject's reach and the monkey did not receive a food reward. Following an incorrect choice, the trial was repeated from the beginning (with the partition placed between experimenter and subject) after a 5-s intertrial interval. The trial was repeated in this fashion until the monkey chose the correct cup and received a food reward. The size of the cup that was used to hide the food was randomized, except that no cup served as the correct choice for more than two consecutive trials. The position of the cup used to hide the food was counterbalanced to eliminate any potential side bias. Subjects completed a minimum of 30 trials with two search locations. Subjects progressed to the MTS phase after finding food on the first trial on $80 \%$ of 30 consecutive trials involving two search locations.

\section{Matching-to-Sample}

After completing preliminary training, subjects were exposed to basic MTS trials. (See Figure 1 for a diagram of the stimulus arrangement for MTS and subsequent testing phases.) For these trials, a pair of cups of different sizes was placed on the lower tier of the platform (hereinafter referred to as the subject's set). A single cup that matched the size of one of these two cups was placed in the center of the higher tier (hereinafter, the experimenter's set). For MTS, cups in the subject's set were randomly assigned to one of five possible positions on the platform, whereas the single cup in the experimenter's set remained fixed in the middle position on the upper tier. Note that for all subsequent testing phases, cups in both the subject's set and the experimenter's set were randomly assigned to one of five positions on each tier of the platform. Therefore, there was no consistent spatial arrangement of the varying-sized cups in each set, and the spatial arrangement of each set was independent of the other. This procedure was adopted so that subjects could not develop a successful strategy on the basis of the spatial position or alignment of stimuli. The manipulation of the cups and the food for all testing phases was done with the partition blocking the subject's view of the platform (as illustrated in Figure 2).

Before the start of a trial, the experimenter hid food under the appropriate cups, out of the subject's view. The experimenter then gained the subject's attention and revealed a food item that had

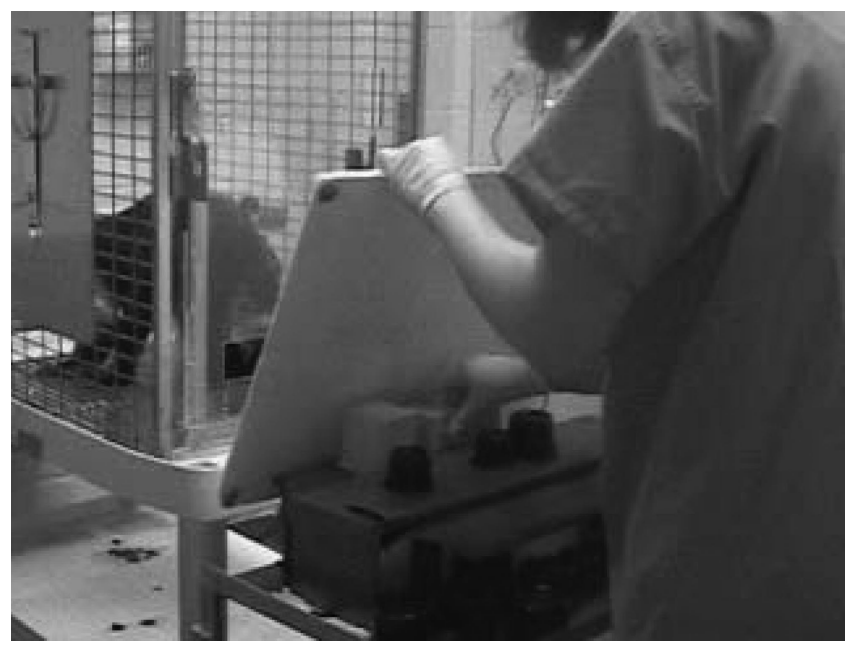

Figure 2. The experimenter places an opaque barrier between the monkey and the experimental apparatus while she baits her cup and the monkey's cup. The photograph also shows the arrangement of stimuli on the experimenter's shelf (above) and the monkey's shelf (below). 
been hidden under the cup on the upper tier. The platform was then pushed within the subject's reach, and the subject was permitted to search under one of the two cups on the bottom tier. To find the hidden food, the subject had to choose the cup that matched the size of the cup under which the food was hidden on the higher tier. (Note: For all testing phases, the higher tier of the stimulus platform was very difficult, but not impossible, for the subjects to reach because of its distance from the testing cage. If a subject managed to reach a cup on the higher tier, this was considered an incorrect response, and the trial was repeated. This happened on fewer than $1 \%$ of trials.) Each subject continued testing until he reached a criterion of 9 correct trials out of 11 consecutive trials in two consecutive testing sessions. This distribution of scores ( 9 out of 11 trials) represents a significant difference $(p<.05)$ in the number of correct responses in relation to the total number of choices made according to the binomial distribution with chance responding of 0.50 .

\section{Relational Matching}

After reaching criterion on the MTS phase, subjects were exposed to relational matching trials. In this phase, all four cups used were different sizes, and a food item was first hidden under one of two cups in the subject's stimulus set and the cup of the same relative size in the experimenter's set, out of the subject's view. At the start of a trial, the experimenter revealed and pointed to the food item hidden under the cup in the experimenter's set with the same size relation (i.e., large or small) to that of the cup hiding the food in the subject's set. To find the hidden food, the subject needed to recognize the relation of the cups in the experimenter's set and use the assessment of this relation to search under the cup of the same relative size in his own set. If the subject made an error on his first search attempt, the trial was repeated until the subject searched in the correct location. Subjects continued testing until they reached a criterion of 9 correct trials out of 11 trials in two consecutive testing sessions or completed 600 trials. If a subject failed to reach criterion after 600 trials, testing was discontinued.

\section{Relational Matching With Distracter}

The procedure in this phase was identical to that used in the relational matching phase. The primary difference between the two phases was that the experimenter's stimulus set and the subject's set contained a cup of identical size that served as a distracter. The cups in the two sets did not, however, share the same size relation. For example, if both sets contained the same size 3 cup, in the experimenter's set this cup served as the largest of the set, whereas in the subject's set this cup served as the smallest. Therefore, if the experimenter hid the food under the largest cup (size 3) in her set, the subject had to ignore the identical cup in his set and choose the largest cup (size 5) based on a relational size rule. Another difference in this phase was that half of the trials involved using two sets of two cups, and half involved using two sets of three cups. In the three-cup sets, the intermediate cup was never the sample.

Trials involving two- and three-cup sets were randomly interspersed within each testing session. The subject continued testing until he reached the criterion of 9 out of 11 trials correct in consecutive testing sessions for the two-cup trials and 7 out of 11 trials correct in consecutive testing sessions for the three-cup trials.

\section{Relational Matching With Distracter: Transfer Test With} Novel Stimuli

Once a subject reached criterion on the relational matching with distracter task, he was exposed to a transfer test involving novel stimuli (yellow cubes). For transfer trials, two sets of yellow cubes were used. As this task was expected to be difficult for subjects, these transfer trials were interspersed randomly with trials from the previous testing phase (relational matching with distracter) using the familiar stimulus set (black cups). As with the previous phase, half of the trials involved sets of two stimuli, and the other half involved sets of three stimuli. As in the previous relational matching task with three cups, the intermediate cup was never correct. Each testing session consisted of four trials involving yellow cubes (transfer trials) and seven trials involving black cups (familiar trials), in random order. The subject completed a total of 60 transfer trials (30 trials involving sets of two stimuli and 30 involving sets of three stimuli).

\section{Relational Matching With Distracter: Transfer Test With Dissimilar Stimuli}

In the final transfer phase, the subject was presented with relational matching with distracter trials that had an additional modification. In this phase, the experimenter's set of stimuli differed in color, shape, and size from the subject's set (see Figure 3). For example, for each trial in a session, the experimenter's set of stimuli consisted of three black cups of varying sizes and the subject's set consisted of three yellow cubes of varying sizes. In this phase, all trials involved sets of three stimuli (or three choices). A correct search required using a relational size rule to match physically dissimilar stimuli (i.e., the largest black cup with the largest yellow cube). The subject was presented with three of these transfer sessions, for a total of 33 trials.

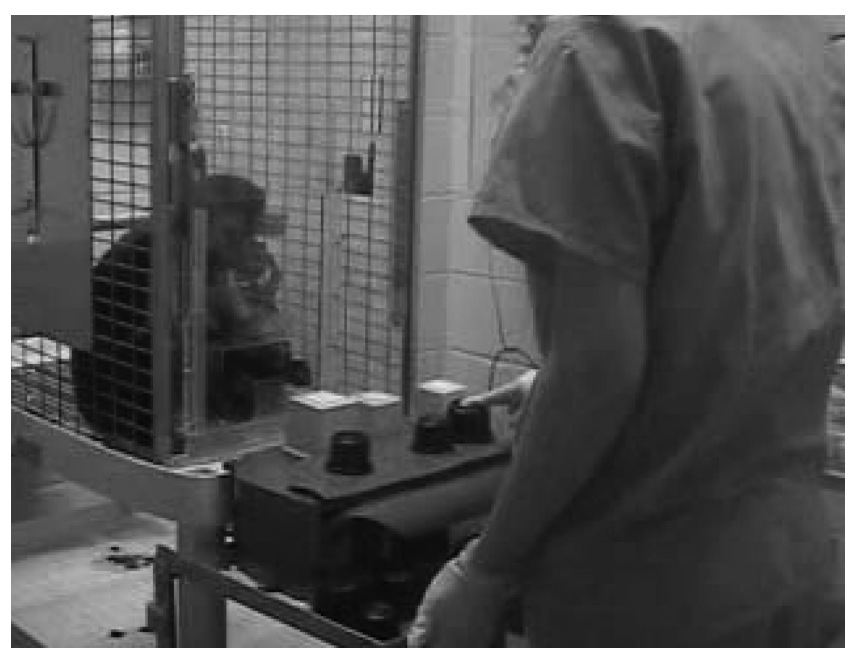

Figure 3. The experimenter places her finger on the baited cup in her array as the monkey watches in the choice phase in the relational matching task with dissimilar stimuli. Note that the experimenter's set is composed of black cylindrical cups and the monkey's set is composed of yellow rectangular cubes. 


\section{Control for Experimenter Cues}

To determine whether subjects were making choices on the basis of inadvertent cues from the experimenter during testing (i.e., gaze direction or body tilting), a second video camera was positioned next to the monkey's testing cage to film the experimenter from the subject's perspective. This additional camera view was used to film the two transfer phases. Following testing, three human observers watched 29 randomly selected trials from these phases from the subject's perspective. The observers were instructed to determine the position of the correct choice (right, middle, or left) solely on the basis of the experimenter's actions as she presented each trial to the subject. The observers (like the subject) were unaware of which cup was baited and were unable to see the cups as the experimenter hid the food. If the observers were unsure of their choice, they were asked to guess a location and indicate that they were not sure of their choice. The observers indicated that they were not sure of their choice on the basis of the experimenter's actions in more than $98 \%$ of the trials scored. The observer's choices were compared with the correct choices for each trial, and binomial tests revealed that the observers chose the correct position at or below chance levels. This indicates that the experimenter provided no reliable cues that humans could detect as to the location of the correct choice other than showing the subject the hidden food in experimenter's stimulus set.

\section{Analysis}

Performance for the matching and relational matching phases was measured in terms of the number of trials required to reach criterion. We considered only first choices when determining whether subjects reached criterion. A binomial test was used to determine whether subjects preferred to choose the stimulus that was the exemplar's physical match if an error was committed in the relational matching with distracter phase, using a chance level of .33 .

Performance for the two relational matching transfer tasks was measured solely in terms of whether the subject committed fewer errors than expected by chance according to a binomial test. It is important to note that the intermediate-sized cup never served as the stimulus in any testing phase. Therefore, only the largest and the smallest cups were possible correct options. Although in one sense, this sets chance responding to .50 (as the subject was choosing between the smallest and largest stimuli), the subject first had to judge the relative size of each cup to identify the largest and smallest, and this required comparing all three cups. For this reason, we felt justified at setting chance performance at .33 for trials involving sets of three stimuli. However, we also analyzed the data using .50 as chance responding, to be conservative. The results did not change whether chance was set at .50 or .33. Alpha was set at .05 for all analyses.

\section{Results}

\section{Matching-to-Sample}

All 4 subjects reached criterion on the MTS task. Subjects required between 309 and 1,113 trials to reach criterion on this task (see Figure 4). Nick was the only monkey of the 4 tested that

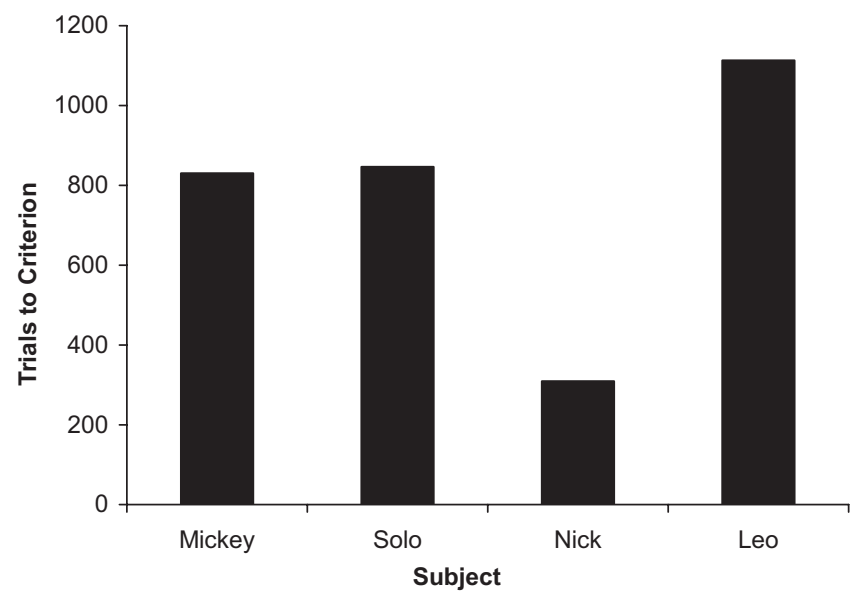

Figure 4. Number of trials required to reach criterion for the matchingto-sample phase.

showed evidence of a side bias for MTS, with $63 \%$ of his choices (202 of 322) being made to his right side.

\section{Relational Matching}

Three monkeys (Nick, Leo, and Solo) failed to reach criterion for the relational matching phase after completing 600 trials and were not tested further. Mickey was the only monkey to reach criterion on this task. He reached criterion on this phase after completing 143 trials.

\section{Relational Matching With Distracter}

Mickey reached criterion on the relational matching with distracter phase after having completed 28 of the two-cup trials and 55 of the three-cup trials. For trials involving sets of three cups, Mickey chose the cup that was not the exemplar's physical match on 46 of 72 trials that resulted in error. A binomial test revealed that when Mickey committed an error, he preferred the cup that was not the physical match to the exemplar at above-chance levels $(p<.05)$.

\section{Relational Matching With Distracter: Transfer Test With Novel Stimuli}

In the first transfer phase, Mickey was presented with familiar relational matching with distracter trials (involving black cups) that were mixed with transfer trials involving novel stimuli of different color, shape, and size (yellow cubes). Mickey searched in the correct location on 38 out of 60 transfer trials, with correct searches occurring on 60\% (18 of 30) of trials involving two search locations $(p=.08)$ and $67 \%$ (20 of 30) of trials involving three search locations $(p<.05)$. Mickey was correct on $86 \%(42$ of 49) of trials involving two search locations and the familiar black cups and 63\% (35 of 56) of trials involving three search locations and black cups. Overall, Mickey made errors on 28 out of $105(27 \%)$ familiar trials with black cups and on 23 out of 60 $(38 \%)$ transfer trials involving the novel yellow cubes. 


\section{Relational Matching With Distracter: Transfer Test With Dissimilar Stimuli}

For the final transfer phase, Mickey was presented with relational matching with distracter trials in which the experimenter's stimulus set and the subject's stimulus set were different from one another (i.e., the experimenter's set was composed of black cups and the subject's set was composed of yellow cubes). All of these trials had three possible search locations (setting chance performance at 33\%). In this phase, Mickey searched in the correct location on $70 \%$ (23 of 33 ) of trials. He chose the correct location on 7 of 11 trials in the first testing session and 8 of 11 on each of the final two sessions. This performance is significantly above chance according to binomial tests.

We conducted additional analyses to examine the errors Mickey made during the final testing phase. Mickey showed no bias in his errors in regard to the relative position of the stimuli. He made four errors to the stimulus on the right, three to the stimulus in the middle, and three to the stimulus on the left. We considered the possibility that Mickey may have made more errors when the exemplar was directly aligned with another stimulus in his set. For example, if the exemplar was in the fourth position in the experimenter's set, Mickey may have been tempted to choose the stimulus directly aligned with this stimulus (in the fourth position in the subject's set). This did not appear to be the case, however, as only four errors involved stimuli that were in direct alignment with the exemplar $(p>.05)$. Nor did Mickey show a bias to choose a stimulus of a particular relative size. Six out of 10 errors involved selecting the intermediate-sized stimulus, three involved the smallest, and one involved the largest. Because Mickey made errors by selecting the intermediate-sized stimulus, this suggests that he was not using a rule that involved simply eliminating the intermediate-sized stimulus and choosing between the remaining two stimuli.

\section{Discussion}

The results of this study provide evidence that a capuchin monkey can solve analogical problems similar to those solved by young children and chimpanzees. Although only 1 subject of the 4 tested reached criterion on the relational matching task with a distracter, he did so after completing relatively few of these trials (143). It was originally believed that the relational matching tasks would prove more difficult in comparison to the physical matching tasks. The relational matching tasks were clearly difficult for the majority of subjects, as was particularly evident in the performance of Nick, who solved the MTS task in relatively few trials (300) but failed to reach criterion after completing 600 trials on the relational matching task. However, the subject who solved the relational matching task (Mickey) did so in many fewer trials than he required to reach criterion on the MTS task (more than 800). It is possible that presentation of the physical matching tasks before the relational matching tasks may have made it more difficult for monkeys to switch to making a choice on the basis of size relations. Alternatively, learning the physical matching rule first may have facilitated relational matching performance. There is evidence of this facilitation (known as the progressive alignment effect) in children who first learn a physical matching rule and are then presented with a relational matching task (Kotovsky \& Gen- tner, 1996). Future studies could examine the effects of presentation order on the development of relational reasoning in monkeys by counterbalancing the order in which these tasks are presented. In any case, although the sample size for this experiment was small, it is apparent that there are individual differences in the ability to solve these problems, and further investigation is necessary to determine the role that the scaffolded presentation of these tasks plays in performance.

The most significant finding of this study was that 1 subject, Mickey, used a relational matching rule to match items from two physically dissimilar sets of stimuli (the final test phase). This phase involved a problem that was most clearly analogical in nature because solving the problem involved assessing the relative size of the exemplar in comparison to the other items within one set, and then using that assessment to choose the stimulus of the same relative size in the subject's set, which consisted of entirely different items. Although it could be argued that Mickey could solve the relational matching with distracter task by excluding the physical match and then choosing the cup that is most similar to the exemplar, this strategy would not result in above-chance performance for the final transfer phase. In this phase, there was no true physical match to exclude (because the items in both sets differed in color, shape, and size), and choosing the stimulus that was the most similar in size to the exemplar would not result in above-chance performance with three choices. Therefore, the results of this final phase of testing provide strong evidence that Mickey was using analogical reasoning to solve this problem.

The only analogical problem in which Mickey did not reach above-chance performance involved sets of two novel stimuli (18 of 30 trials correct vs. 20 of 30 trials correct for three-cup trials). Although the difference in the number of correct choices between two- and three-stimulus trials is small, it was enough to affect statistical probability. Additional testing is necessary to determine whether the difference is reliable. Regardless of his performance on this testing phase, Mickey solved two out of three transfer tests at above-chance levels, including the final transfer phase that represented the most complex analogical problem in this series of tasks.

Mickey's performance provides compelling evidence that a member of a New World monkey species can solve a problem involving analogical reasoning. This finding suggests that there is no firm divide between apes and monkeys in their capacity to reason in this way. Gentner et al. (1995) has argued that analogical reasoning abilities in children develop as a function of increasing general knowledge of the world rather than as a function of age. We would argue that prior experience solving a wide range of problems facilitated the analogical reasoning abilities of the nonhuman primates that have shown the ability to complete these tasks. The chimpanzees that have solved these problems, and the capuchins in the current study, have had years of experience solving a wide range of experimental problems including threedimensional search tasks. As there is much evidence to suggest that prior knowledge plays a key role in problem solving in humans (i.e., Alexander \& Schwanenfluegel, 1994; Gaultney et al., 1992; Schneider, Bjorklund, \& Maier-Bruckner, 1996; Woloshyn et al., 1992), it is possible that the knowledge gained from the exposure to other similar tasks aided these nonhuman primates to reason analogically. In addition, the scaffolded nature of this set of tasks was designed to aid in the development of the use of a 
relational rule. It would be useful to examine the extent to which experience specific to this task affects performance in comparison with general problem-solving experience gained outside of this task by presenting experimentally naïve monkeys with this same set of problems. If naïve monkeys are able to solve this problem with the help of this scaffolded set of tasks, this would suggest that experience specific to this problem plays a greater role in facilitating the use of relational rules than does more general problemsolving experience.

Mickey's singular success in this series of tasks begs the question of whether he is generally good at solving cognitive tasks. This is not the case, however, as Mickey's prior performances on other experimental tasks were decidedly mediocre in relation to the other capuchins in our lab. What distinguished him from the others in this experiment was his relatively high motivation and attention to the task. It is not clear why this particular task appealed to him more than others presented in our lab, although it is possible that Mickey showed greater motivation in this task because it involved actively searching for food. It remains unclear, however, why Mickey performed better on this task in comparison with the three other monkeys tested.

The findings of the current study correspond with those of Spinozzi et al. (2004), who demonstrated that capuchins could match images on the basis of abstract spatial relations (above and below). Although there are similarities between the Spinozzi et al. study and the current study, we believe that the methodology used in the current study allows for a more direct comparison of analogical tasks performed by children and chimpanzees and therefore allows for a more accurate comparative perspective. We would also argue that the analogical task solved by Mickey in the current study was more challenging than the spatial task performed by the monkeys in Spinozzi et al.'s study for two primary reasons. First, the subjects in the current study were required to direct their attention to the location of hidden food that was unattainable (in the experimenter's stimulus set) and use this information to search under the object of analogous size in their own set. Attending to a series of events (i.e., seeing hidden food revealed that is out of reach and using the location of this hidden food to search in the analogous location) is arguably more difficult than a judgment made during viewing concurrent displays. Moreover, previous research with chimpanzees and monkeys has suggested that monkeys and apes experience initial difficulties in using cues given by an experimenter to find a hidden object (Call, Agnetta, \& Tomasello, 2000; Vick \& Anderson, 2000). A second difference between Spinozzi et al.'s task and the current study was the number of stimuli the subjects had to choose from. Spinozzi et al.'s task involved only binary choices, whereas the current study presented three choices as well as binary choices. In the final transfer phase, Mickey was presented with three cups of different sizes that made up the sample set and a choice set that was also composed of three stimuli of different sizes. There is a reduced probability of guessing the correct choice in the three-choice task (chance $=.33$ ) compared with the binary task (chance $=.50$ ), and the threechoice task also requires that the subject assess the relative size of a greater number of objects. For these reasons, it can be argued that this task represents a more difficult analogical problem.

Despite the differences between these two studies, both suggest that some degree of relational reasoning is present in a species other than apes and humans. As analogical reasoning greatly increases the efficiency of problem solving, it would be expected that the rudiments of this kind of reasoning would be found in a variety of species, not limited to primates. In support of this hypothesis, a series of relational matching studies involving twodimensional arrays of items has suggested that the rudiments of analogical reasoning may be present in pigeons (Castro \& Wasserman, 2007; Wasserman, Frank, \& Young, 2002). In light of increasing evidence that analogical reasoning may not be limited to a very short list of animals, it is important to continue testing a diverse variety of species to gain a better understanding of the origins of analogical thought.

Although Mickey required a relatively large number of trials to learn the MTS task (more than 800), he reached criterion on the other tasks involving use of a relational rule in relatively few trials (150 or less). His rapid progression through the testing series after mastering the MTS rule may have been a result of our use of a scaffolded series of matching tasks (MTS followed by relational matching, followed by relational matching with distracter, etc.). Previous studies have not presented monkeys with analogical problems in this scaffolded way. Clearly, replication of our study is necessary, and future studies should directly compare the performance of monkeys presented with analogical problems in this manner to the performance of monkeys that are required to solve the problem with no other prior problem-solving experience. In addition, a direct comparison between the performance of chimpanzees and capuchins on this task would be particularly useful. It would also be useful to attempt to use variations of this methodology to study other nonhuman primates (and nonprimate species), as analogical reasoning may be a cognitive ability that is more widespread than was once believed.

\section{References}

Alexander, J. M., \& Schwanenfluegel, P. (1994). Strategy regulation: The role of intelligence, metacognitive attributions, and knowledge base. Developmental Psychology, 30, 709-723.

Anderson, J. R. (2000). Cognitive psychology and its implications (5th ed.). New York: Worth.

Call, J., Agnetta, B., \& Tomasello, M. (2000). Cues that chimpanzees do and do not use to find hidden objects. Animal Cognition, 3, 23-34.

Castro, L., \& Wasserman, E. A. (2007, March). Can a pigeon learn to complete an analogy? Poster presented at the 14th International Conference on Comparative Cognition, Melbourne, FL.

Cummins-Sebree, S., \& Fragaszy, D. (2005). Choosing and using tools: Capuchins (Cebus apella) use a different metric than tamarins (Saguinus oedipus). Journal of Comparative Psychology, 119, 210-219.

Fragaszy, D., \& Cummins-Sebree, S. (2005). Relational spatial reasoning by a nonhuman: The example of capuchin monkeys. Behavioral Cognitive NeuroscienceReviews, 4, 282-306.

Fragaszy, D. M., Johnson-Pynn, J., Hirsh, E., \& Brakke, K. (2003). Strategic navigation of two-dimensional alley mazes: Comparing capuchin monkeys and chimpanzees. Animal Cognition, 6, 149-160.

Gaultney, J., Bjorklund, D. F., \& Schneider, W. (1992). The role of children's expertise in a strategic memory task. Contemporary Educational Psychology, 17, 244-257.

Gentner, D. (1977). Children's performance on a spatial analogies task. Child Development, 48, 1034-1039.

Gentner, D., \& Rattermann, M. J. (1991). Language and the career of similarity. In S. Gelman \& J. Byrnes (Eds.), Perspectives on language and thought: Interrelations in development (pp. 225-277). Cambridge, England: Cambridge University Press. 
Gentner, D., Rattermann, M. J., Markman, A., \& Kotovsky, L. (1995). Two forces in the development of relational similarity. In T. Simon \& G. Halford (Eds.), Developing cognitive competence: New approaches to process modeling (pp. 263-313). Hillsdale, NJ: Erlbaum.

Gillian, D. J., Premack, D., \& Woodruff, G. (1981). Reasoning in the chimpanzee: I. Analogical reasoning. Journal of Experimental Psychology: Animal Behavior Processes, 7, 1-17.

Hoy, E. A., \& Fragaszy, D. (2004, June). The effects of experience and presentation order on the ability of capuchin monkeys (Cebus apella) to plan their actions when solving two-dimensional detour problems. Poster presented at the conference for the American Society of Primatologists, Madison, WI.

Johnson-Pynn, J., Fragaszy, D. M., Hirsh, E., Brakke, K., \& Greenfield, P. (1999). Strategies used to combine seriated cups by chimpanzees (Pan troglodytes), bonobos (Pan paniscus), and capuchins (Cebus apella). Journal of Comparative Psychology, 113, 137-148.

Kotovsky, L., \& Gentner, D. (1996). Comparison and categorization in the development of relational similarity. Child Development, 67, 27972822.

Leighty, K. A., \& Fragaszy, D. M. (2003). Joystick acquisition in tufted capuchins (Cebus apella). Animal Cognition, 6, 141-148.

Oden, D. L., Thompson, R. K. R., \& Premack, D. (2001). Can an ape reason analogically? Comprehension and production of analogical problems by Sarah, a chimpanzee (Pan troglodytes). In D. Gentner, K. Holyoak, \& B. Kokinov (Eds.), Analogy: Theory and phenomena (pp. 471-497). Cambridge, MA: MIT Press.

Piaget, J. (1966). The genetic approach to the psychology of thought. Journal of Educational Psychology, 52, 275-281.

Premack, D., \& Premack, A. (2003). Original intelligence: Unlocking the mystery of who we are. New York: McGraw-Hill.

Rattermann, M. J., Gentner, D. \& DeLoache, J. (1987, April). Young children's use of relational similarity in a transfer task. Poster presented at the biennial meeting of the Society for Research in Child Development, Baltimore, MD.

Rosengart, C. R. (2005). A dynamic account of the A-not-B error in capuchin monkeys (Cebus apella). Unpublished doctoral dissertation, University of Georgia, Athens.
Rosengart, C. R., \& Fragaszy, D. M. (2005). Experience and materials affect combinatorial construction in capuchin monkeys (Cebus apella). Journal of Comparative Psychology, 119, 166-178.

Schneider, W., Bjorklund, D., \& Maier-Bruckner, W. (1996). The effects of expertise and IQ on children's memory: When knowledge is, and when it is not enough. International Journal of Behavioral Development, 19, 773-796.

Spinozzi, G., Lubrano, G., \& Truppa, V. (2004). Categorization of above and below spatial relations by tufted capuchin monkeys (Cebus apella). Journal of Comparative Psychology, 118, 403-412.

Thompson, R. K. R., \& Oden, D. L. (1995). A profound disparity revisited: Perception and judgments of abstract identity relations by chimpanzees, humans infants, and monkeys. Behavioural Processes, 35, 149-161.

Thompson, R. K. R., Oden, D. L., \& Boysen, S. T. (1997). Language-naïve chimpanzees (Pan troglodytes) judge relations between relations in a conceptual matching-to-sample task. Journal of Experimental Psychology: Animal Behavior Processes, 23, 31-43.

Thompson, R. K. R., Oden, D., Hyle, M., Hoy, E., Rapuano, M., \& Safro, J. (2000, November). Group habituation/dishabituation by capuchin monkeys to objects and relations. Paper presented at the 41st Annual Meeting of the Psychonomic Society, New Orleans, LA.

Vick, S., \& Anderson, J. R. (2000). Learning and limits of use of eye-gaze by capuchin monkeys (Cebus apella) in an object-choice task. Journal of Comparative Psychology, 114, 200-207.

Wasserman, E. A., Frank, A. J., \& Young, M. E. (2002). Stimulus control by same-versus-different relations among multiple visual stimuli. Journal of Experimental Psychology: Animal Behavior Processes, 28, $347-$ 357.

Woloshyn, V., Pressley, M., \& Schneider, W. (1992). Elaborativeinterrogation and prior-knowledge effects on learning of facts. Journal of Educational Psychology, 84, 115-124.

Received June 8, 2007 Revision received January 28, 2008 Accepted February 6, 2008 